\title{
Tumores de vejiga pTO tras cistectomía radical: análisis de nuestra serie
}

\author{
Mallén Mateo E, Gil Martínez P, Gil Sanz MJ, Sancho Serrano C, Pascual Regueriro D, \\ Rioja Sanz LA.
}

Servicio de Urología. Hospital Universitario Miguel Servet. Zaragoza.

Actas Urol Esp. 2006;30(8):763-771

\section{RESUMEN}

TUMORES DE VEJIGA PTO TRAS CISTECTOMÍA RADICAL: ANÁLISIS DE NUESTRA SERIE

Objetivos: Estudiar los datos de progresión y supervivencia en los 43 pacientes cistectomizados y catalogados de pT0 según la clasificación TNM-2002.

Material y Métodos: Entre 1988 y 2003 se han realizado en nuestro centro 420 cistectomías, en 43 casos(10,2\%) no se halló tumor en la pieza anatomopatológica siendo éstos el núcleo de nuestro análisis.

Resultados: En estos 43 casos el estadio clínico inicial (en la resección transuretral de tumor vesical previa a la cistectomía) fue $\mathrm{T} 1$ en 10 casos $(23,3 \%)$, T2 en 31 casos $(72 \%)$ y T3 en 2 casos. En cuanto al grado 24 pacientes presentaron G2 (55,8\%) y $19(44,2 \%)$ fueron G3. La mediana entre la de resección transuretral de tumor vesical diagnóstica (RTU de TM vesical) y la cistectomía fue de 44 dias y la mediana del seguimiento fue de 89,3 meses.

Progresión. La supervivencia libre de progresión en los 43 pacientes fue de 180,6 meses, pero durante el seguimiento apareció progresión en 7 pacientes con una mediana de supervivencia libre de progresión desde la fecha de la cistectomía de 36 meses (3-126).

Supervivencia cáncer-específica (SCE). Durante el seguimiento, 5 enfermos fallecieron, cuatro por la enfermedad y el quinto por un cáncer de pulmón. Si analizamos la SCE, según la anatomía patológica de la RTU de tumor vesical previa, para los T2 la media de SCE es de 180 meses, disminuyendo considerablemente para T3 hasta 35 meses. Similar ocurre con el grado de diferenciación tumoral, disminuyendo significativamente a medida que avanza el grado, con una media de SCE para los G3 de122,6 meses. Del mismo modo ocurre con la afectación ganglionar en la pieza de la cistoprostatectomía radical, con una SCE de 188 meses cuando es NO y de 54 meses si los ganglios son positivos ( $\mathrm{N}+$ ).

Conclusiones: En nuestra experiencia los tumores uroteliales pTO presentan un periodo libre de enfermedad prolongada (mediana de 180 meses).

Los factores de riesgo asociados a un menor periodo libre de enfermedad (PLE) son alto grado de diferenciación (G3, 116 meses), la infiltración de capas profundas en la RTU de tumor vesical(T3, 32 meses) y la afectación ganglionar (pN+) 45 meses.

Palabras clave: Cistectomía. Tumor vesical infiltrante. pTO.

\section{ABSTRACT}

STAGE PTO CYSTECTOMY: A REVIEW OF OUR SERIE

Objectives: To evaluate the data of progression and survival in 43 patients who underwent cystectomy with stage pTO according to classification TNM-2002.

Matherials and methods: between 1988 and 2003 in our center had realized 420 cystectomies, 43 patients (10.2\%) had not tumor in the cystectomy specimen.

Results: In these 43 cases the initial clinical stage (in the transuretral resection of bladder) was T1 in 10 cases (23,3\%), T2 in 31 cases $(72 \%)$ and T3 in 2 cases. As far as the degree 24 patients presented G2 (55.8\%) and 19 (44.2\%) were G3.

Median time from the transuretral resection to the cistectomy was of 44 days at a median follow-up of 89.3 months. Progressionfree survival in the 43 patients was of 180,6 months, but during the follow-up it appeared progression in 7 patients, with disease free survival at 36 months (3-126) ,since the date of the cistectomía.

During the follow up, 5 patients died. When we analyzed the cancer-specific survival according to tumor stage, for the T2 with an average cancer-specific survival is of 180 months, decreasing to 35 months considerably for T3. Similar it happens with the degree of differentiation, significantly diminishing as it advances the degree, with an average of cancer-specific survival for the G3 at 122.6 months. In the same way it happens with pathological positive lymph nodes in the radical cistectomy , with a cancer-specific survival of 188 months when it is NO and of 54 months if the adenopathy was positive (N+).

Conclusion: In our experiencie urothelial carcinoma pT0 present a prolonged free period of disease (medium of 180 months). The associated factors of risk to a smaller free period of disease are high degree of differentiation (G3, 116 months), the infiltration of deep layers in the transuretral resection(T3, 32 months) and the ganglionary affectation (pN+ 45 months).

Keyword: Cystectomy. Bladder cancer. pTO. 
$\mathrm{E}^{1}$ cáncer vesical es uno de los tumores más recuentes en el hombre, ocupa el segundo lugar dentro de los tumores urológicos, después del de próstata, comportando una gran morbimortalidad. Se estima que el cáncer vesical supone el $2 \%$ de los tumores malignos. Pero sólo el 15-30\% de estos tumores progresan. Los principales factores predictivos de supervivencia y de control del cáncer vesical son la extensión de la enfermedad y el aspecto histológico ${ }^{1}$. Aún siendo de tan suma importancia el carcinoma vesical músculoinvasivo, por su trascendencia clínica, no es muy habitual hallar en la literatura referencias al mismo en los estadios pTO y son series cortas las que podemos encontrar.

Como veremos, no es infrecuente encontrarnos con el diagnóstico del anatomopatólogo de estadio pTO en la pieza de cistoprostatectomía radical, indicada por el resultado de la resección transuretral de tumor vesical previa (RTU de tumor vesical). Esto ocurre en pacientes en los que no hay invasión de la capa muscular o la resección de la tumoración intravesical ha sido completa $^{2}$.

En este estudio queremos analizar los datos de progresión y supervivencia en 43 pacientes cistectomizados y catalogados de pTO según la clasificación TNM 2002. Es un estudio meramente descriptivo, no es objeto de este análisis cuestionar el tratamiento más adecuado para el tumor vesical infiltrante.

\section{MATERIAL Y MÉTODOS}

Hemos realizado un estudio retrospectivo analizando las 420 cistectomías realizadas en nuestro centro entre 1988 y 2003. En 43 casos (10\%) no se halló tumor en la pieza anatomopatológica (pT0) siendo éstos el objetivo de nuestro análisis.

La mayoría de los pacientes presentó hematuria como síntoma fundamental para el diagnóstico, se confirmó la existencia del tumor con métodos de imagen y/o cistoscopia. La metodología de imagen utilizada fue la ecografía y UIV para el diagnóstico inicial, el TAC y la gammagrafía ósea se realizaron en el estudio de diseminación tras el diagnóstico de tumor vesical infiltrante.

Las indicaciones de cistectomía en nuestro centro son por un lado, la invasión de las capas profundas de la vejiga, o en los superficiales de alto riesgo (CIS, T1G3) si no se logra remisión completa tras el segundo ciclo de inducción con $\mathrm{BCG}$, o bien si existe una recidiva precoz en un intervalo menor de 6 meses desde la RTU inicial o si aparece progresión del estadio ${ }^{3,4}$. También está contemplada su realización en aquellos casos en los que el tumor, por su magnitud, su tratamiento sería inabarcable endoscópicamente. Dentro de la técnica quirúrgica utilizada en la cistoprostatectomía radical, en todos los casos se realizó por vía transperitoneal y siempre se llevó a cabo linfadenectomía ileoobturatriz bilateral.

El análisis estadístico ha sido realizado utilizando el programa estadístico SPSS v11, analizando la supervivencia con el método Kaplan Meier.

\section{RESULTADOS}

La edad media de los pacientes fue de 60,3 años (DE: 9,7), 39 pacientes fueron varones y 4 mujeres.

La mediana entre la resección de tumor vesical diagnóstica y la cistectomía fue de 44 días. Y la mediana del seguimiento en estos pacientes fue de 89,3 meses.

Si observamos en esto 43 casos el estadio clínico que nos da la RTU de tumor vesical previa a la cistectomía, fue $\mathrm{T} 1$ en 10 casos $(23,3 \%) \mathrm{T} 2$ en 31 casos $(72 \%)$ y T3 en 2 casos. En cuanto al grado, 24 pacientes presentaron G2 (55,8\%) y 19 fueron G3 (44,2\%). Si analizamos la indicación de cistectomía en los $\mathrm{T} 1$, vemos que en 4 casos fueron T1G3 que no respondieron al tratamiento con BCG, en un caso además se acompañaba de CIS en las biopsias múltiples de control. En los restantes seis pacientes fueron $\mathrm{T} 1 \mathrm{G} 2$, en todos los casos había antecedente de varias resecciones transuretrales previas, entre 5-7 RTU previas, y en dos de estos seis hubo progresión del tumor vesical superficial (TaG1). Eran tumores múltiples, de gran tamaño y recidivantes, en los que el tratamiento endoscópico era insuficiente. Estos casos de $\mathrm{T} 1$ fueron sometidos tras las resecciones transuretrales a tratamiento endovesical, con BCG en 6 casos, Tiotepa en dos y mitomicina C en los dos restantes.

Dentro de los 43 pacientes, se realizaron varios tipos de derivaciones urinarias con sus respectivas reimplantaciones ureterales, la deri- 
vación urinaria más frecuentemente realizada en nuestro centro fue la neovejiga tipo Hautmann, en 29 pacientes. Al principio de nuestra serie se comenzó realizando en estos casos la reimplantación ureteral según técnica de Le-Duc. Actualmente en el Hautmann se realizan dos técnicas, según el cirujano, Wallace II o Abol-Eneim. La segunda derivación en frecuencia fue el Bricker, en siete casos, con derivación Wallace II en todos los casos. En el resto se realizó Camey II, en tres casos (y los tres fueron realizados en la década de los 80) y casos excepcionales de conducto colónico, ureterosigmoidostomía, Mainz I y vejiga padoana.

$\mathrm{Si}$ analizamos las complicaciones intraoperatorias, no hubo grandes complicaciones que reseñar, no hubo que lamentar ninguna muerte intraoperatoria. La complicación más común fue el sangrado intraoperatorio; $27(62,7 \%)$ pacientes requirieron transfusión intraoperatoria por parte del anestesista. La mayoría, 12 pacientes, precisaron 2 concentrados de hematíes, 8 de ellos necesitaron tres bolsas; a tres enfermos se les trasfundió hasta cuatro bolsas y los cuatro restantes sólo fue necesario un concentrado de hematíes. Respecto a las complicaciones del postoperatorio inmediato encontramos infección de la herida quirúrgica en dos casos, íleo paralítico, eventración de laparotomía en tres casos, neumonía intrahospitalaria, un caso de fuga en la anastomosis ureteroileal en una neovejiga tipo Hautmann, que se solucionó con derivación renal, con una nefrostomía. En otro paciente la fuga se diagnosticó en la anastomosis uretral, que cerró con la permanencia de la sonda 5 semanas, cuando lo habitual en nuestro centro es mantener la sonda 3 semanas. Un paciente con neovejiga tipo Hautmann sufrió una complicación con la aparición de una fístula en la bolsa ileal, teniendo que reconvertir a Bricker, en este caso intraoeperatoriamente ya hubo complicaciones, ya que los mesos intestinales eran cortos y gruesos dando pocas facilidades para la elaboración de la neovejiga.

De mayor trascendencia fueron los problemas a medio largo plazo, a reseñar la más frecuente la litiasis en neovejiga, en 7 casos, 6 eran neovejiga tipo Hautmann y en un caso fue en un Camey. En todos ellos se solucionó con litotricia endoscópica.
Las estenosis más frecuentes se dieron a nivel uretrointestinal, en 4 casos en neovejiga tipo Hautmann, precisando en un caso resección y en tres fue suficiente con cuchillete de Sachse para solucionar la estenosis. En dos casos, en un Bricker y en la vejiga Padoana la estenosis fue en la anastomosis ureteral, requiriendo en los dos casos dilatación endoscópica.

Posteriormente a la cistectomía estos pacientes son sometidos a seguimiento oncológico según el protocolo basado en las EAU Guidelines $2001^{3}$. Durante los tres primeros años son sometidos a un primer control al 1,5 mes del alta y posteriormente cada semestre con un estudio bioquímico (con gasometría venosa), ecografía abdominal, Rx simple, urocultivo, y citologías; la Rx. Tórax y UIV la realizamos anualmente. Al cuarto año, si los controles son normales, pasamos a revisiones anuales, y la UIV sólo la realizaremos en casos excepcionales, individualizando los pacientes, cuando el urólogo lo considere oportuno, ej: CIS multifocal, tumor en uréter distal... ${ }^{5}$.

A pesar de resultar pTO en la pieza, en 5 casos $(11,6 \%)$ se encontró afectación ganglionar en la linfadenectomía realizada en la cistectomía. En tres casos el estadio de la RTU de tumor vesical previa fue T2G3, en otro paciente se le había indicado la intervención con un estadio T1G3 y un último con T3bG3. Todos ellos tienen como denominador común el alto grado de indiferenciación tumoral. De los cinco pacientes, tres recibieron quimioterapia adyuvante basada en CMV(cisplatino, metrotexate y vinblastina). De los cinco murieron dos, éstos no habían recibido tratamiento quimioterápico, por la gran afectación del estado general.

Si analizamos la progresión, la supervivencia libre de progresión en los 43 pacientes fue de 180,6 meses, pero durante el seguimiento apareció progresión en 7 pacientes. Cuatro de ellos, con metástasis a distancia y tres con metástasis ganglionares regionales y en uno de éstos además con recidiva pelviana, con una mediana de supervivencia libre de progresión desde la fecha de la cistectomía de 36 meses (3-126). De estos siete pacientes, cuatro recibieron quimioterapia; en el paciente con recidiva pelviana se le realizó nefroureterectomía derecha con resección de pastilla de neovejiga asociando a la quimioterapia, 
cobaltoterapia. La quimioterapia recibida consintió en tres ciclos de CMV. Todos terminaron todos los ciclos, sólo un caso presentó toxicidad hematológica. En dos casos el estado general de los pacientes no permitió añadir tratamiento adyuvante.

En las figuras podemos observar detalladamente la progresión según el estadio, la media de progresión para los T2 es de 162 meses, disminuyendo hasta 32 meses en los T3 (Fig. 1). Similar ocurre con el grado (Fig. 2), cuanto más

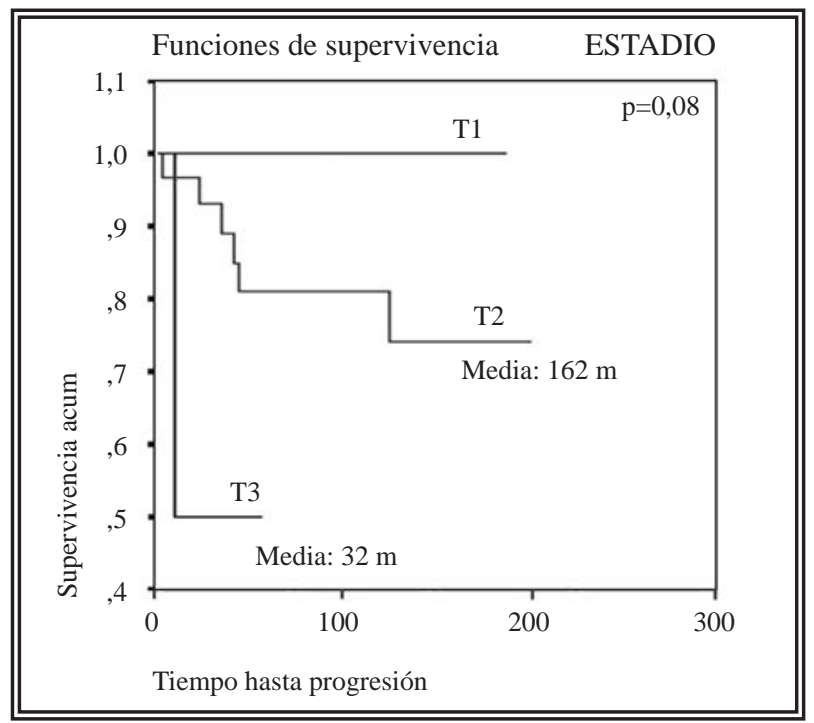

FIGURA 1. Las curvas de Kaplan-Meier muestran el tiempo hasta la progresión según el estadio, grado y afecta ción ganglionar.

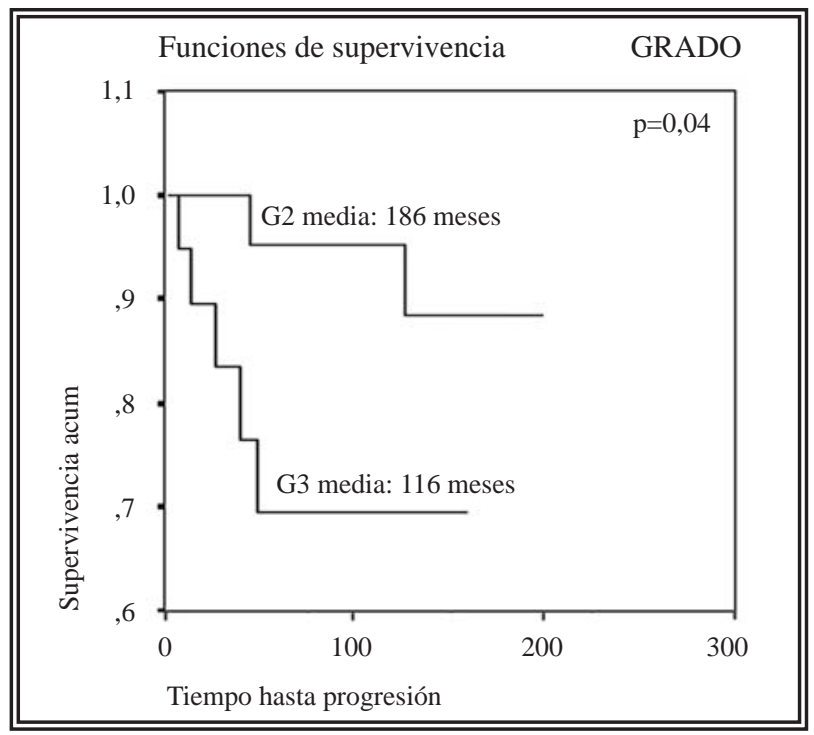

FIGURA 2. Las curvas de Kaplan-Meier muestran el tiempo hasta la progresión según el estadio, grado y afectación ganglionar. indiferenciado (G3) menor es el tiempo hasta la progresión, 116 meses, en contraste con los G2 con 186 meses. Estadísticamente significativa es la diferencia en el tiempo hasta la progresión cuando hay afectación ganglionar, con una media de 179 meses en los PNO y de 45 meses cuando los ganglios son positivos (Fig. 3).

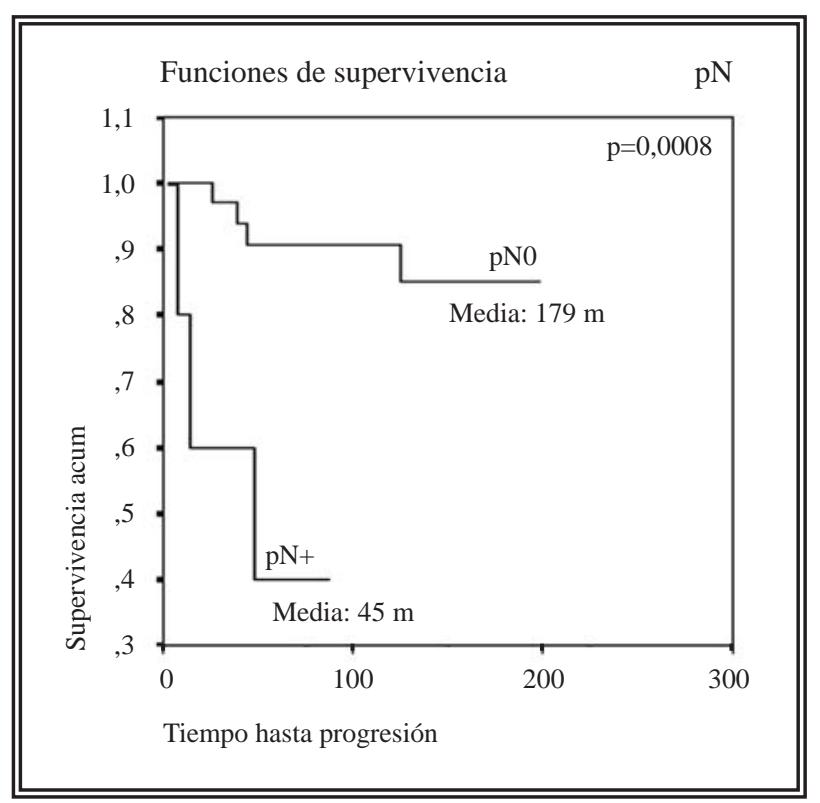

FIGURA 3. Las curvas de Kaplan-Meier muestran el tiempo hasta la progresión según el estadio, grado y afectación ganglionar.

En cuanto a la supervivencia cáncer-específica (SCE), durante el seguimiento, 5 enfermos fallecieron, 4 con metástasis y debido a su neoplasia y un quinto por un cáncer de pulmón. Si analizamos la SCE, según la anatomía patológica de la RTU previa, para los T2 la media de SCE es de 180 meses, disminuyendo considerablemente para T3 hasta 35 meses (Fig. 4).

Similar ocurre con el grado de diferenciación tumoral, disminuyendo significativamente a medida que avanza el grado, con una media de SCE para los G3 de122,6 meses (Fig. 5). Del mismo modo ocurre con la afectación ganglionar en la pieza de la CPR, con una SCE de 188 meses cuando es NO y de 54 meses si los ganglios son positivos (N+) (Fig. 6).

\section{DISCUSIÓN}

Ya hemos mencionado anteriormente las directrices e indicaciones de cistectomía en nuestro centro, que son: la invasión de las capas profun- 


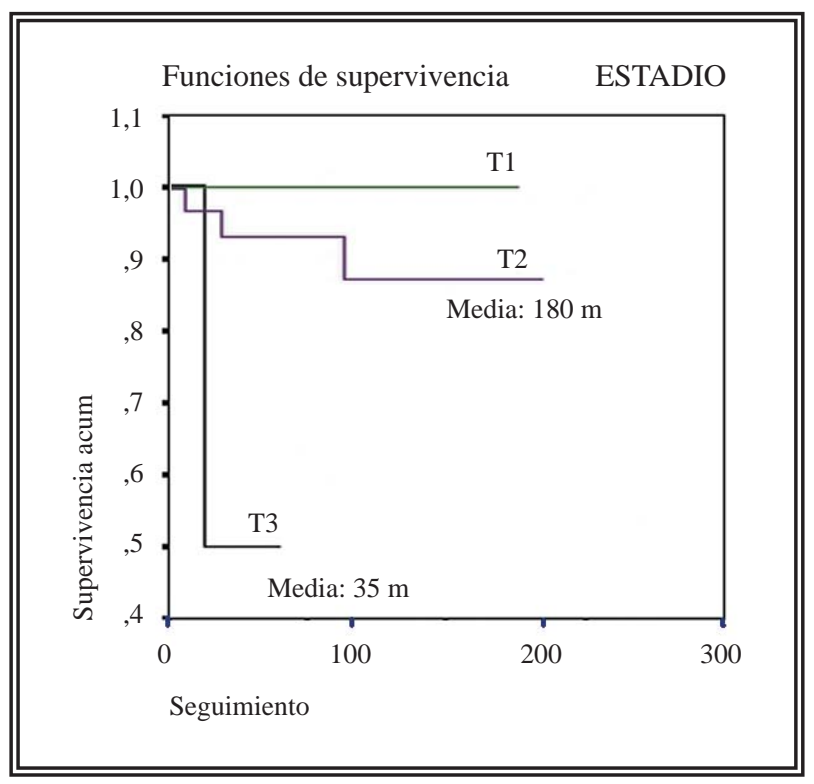

FIGURA 4. Las curvas de Kaplan-Meier muestran las diferencias en la supervivencia cáncer especifica según el estadio, grado y afectación ganglionar.

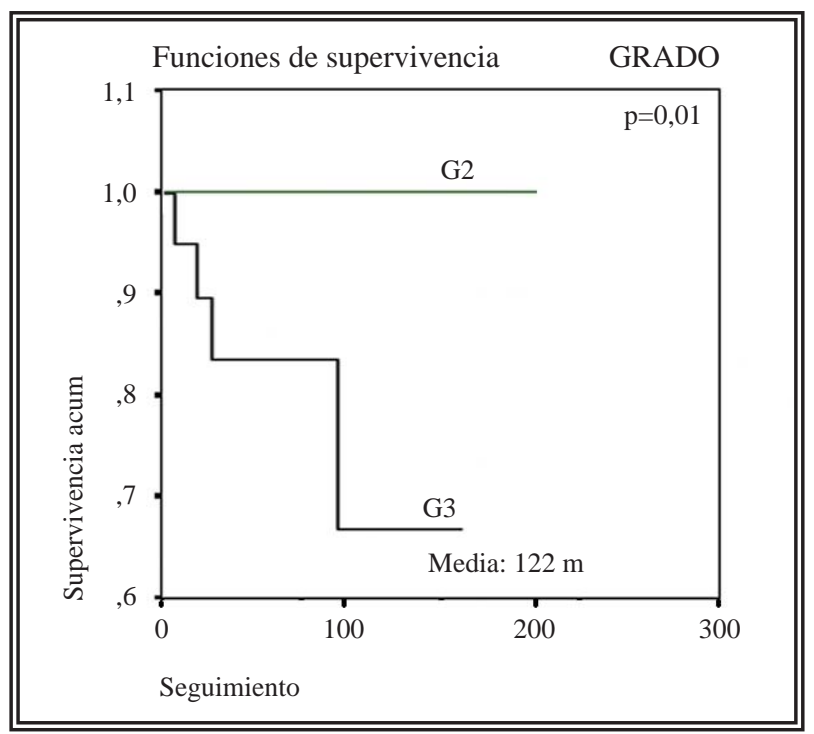

FIGURA 5. Las curvas de Kaplan-Meier muestran las diferencias en la supervivencia cáncer especifica según el estadio, grado y afectación ganglionar.

das de la vejiga, y en los superficiales de alto riesgo (CIS, T1G3) si no se logra remisión completa tras el segundo ciclo de inducción con BCG, o bien si existe una recidiva precoz en un intervalo menor de 6 meses desde la RTU inicial o si aparece progresión del estadio ${ }^{3,4}$. También está contemplada su realización en aquellos casos en los que el tumor superficial por su tamaño, es inabarcable endoscópicamente.

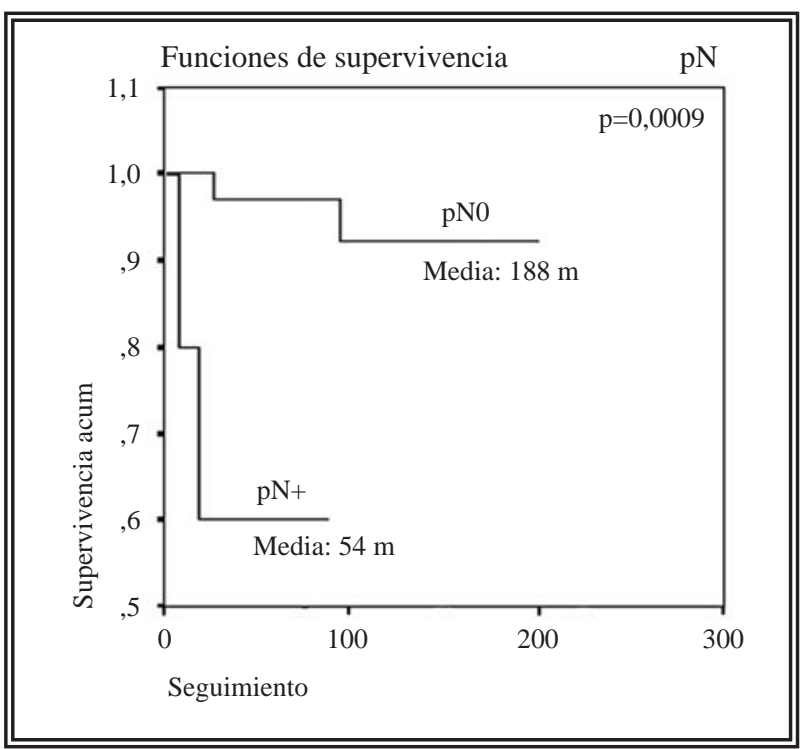

FIGURA 6. Las curvas de Kaplan-Meier muestran las dife rencias en la supervivencia cáncer específica según el estadio, grado y afectación ganglionar.

En cuanto a RTU conservadora, nuestra serie es corta pero también está contemplada según las indicaciones de Solsona ${ }^{6}$. Solamente está indicada en los T2, para ello es necesario realizar biopsias frías de grasa perivesical sin afectación de las mismas. En cuanto al grado, no es criterio de exclusión del protocolo de RTU radical. Se tiene en cuenta el tamaño en tumores sesiles, debe de ser menor de $3 \mathrm{~cm}$.

Se lleva a cabo una resección completa del tumor, llegando a grasa en toda su extensión.

Se diferencian tres muestras dentro de la resección de la tumoración: porción exofítica del tumor, porción endofitica y lecho muscular (muscular propia). Además se deben de tomar varias muestras de biopsias frías de la grasa perivesical. Si éstas se encuentran afectadas por el tumor vesical se excluye la resección tumoral indicando la cistectomía. Dentro del protocolo de RTU radical se realiza biopsias vesicales múltiples, en el mismo acto quirúrgico, para investigar CIS. En este caso, cuando se da la coexistencia con CIS, no es contraindicación de cirugía conservadora ,al programa de RTU radical se añade el tratamiento estándar con BCG.

Pueden darse casos excepcionales, en los que haya afectación del lecho muscular por el tumor vesical (y biopsia fría de grasa negativa) se le da opción al paciente para que elija entre seguir con 
el protocolo de conservación vesical con tres ciclos de quimioterapia adyuvantes a la RTU radical, basados en cisplatino o la realización de cistectomía radical.

Lógicamente en estos casos, en los que se realiza RTU radical, el seguimiento oncológico es más estricto, con resección de la cicatriz al tercer y sexto mes, junto con biopsias múltiples de la vejiga y citologías cada cuatro meses, realización de TAC y Rx. Tórax cada 6 meses $^{6}$.

En los pacientes con cáncer vesical sometidos a cistectomía radical el esquema de seguimiento irá orientado a la detección de: la recidiva local en la pelvis, la aparición de nuevos tumores en el urotelio restante y en los segmentos intestinales utilizados para la derivación, la aparición de metástasis a distancia, y el diagnóstico precoz de las posibles complicaciones y efectos secundarios de la derivación urinaria ${ }^{7}$. La recidiva local es más frecuente en los tumores localmente avanzados (pT3b-pT4a) sin que su frecuencia se modifique por la administración de radioterapia preoperatoria. Los avances de los métodos diagnósticos y en la técnica quirúrgica utilizada han reducido la frecuencia de la recidiva local, desde el $37 \%$ descrito en los años 50 hasta el 3,9\%-18\% en la actualidad ${ }^{8,9}$.

La recidiva local suele diagnosticarse como media entre los 8,3 y los 13,5 meses después de la cistectomía con un intervalo entre 1 y 79 meses, lo que parece confirmar que se debe a tumor residual microscópico en la pelvis o en los ganglios linfáticos. Aproximadamente la mitad de los pacientes están asintomáticos en el momento del diagnóstico de la recidiva local. La supervivencia a medio plazo de estos pacientes suele ser excepcional, comunicándose supervivencias medias, a pesar del tratamiento de rescate, que oscilan entre los 4 y los 9 meses con un intervalo entre 1 y 58 meses después del diagnóstico de la recidiva local. Debido a esta corta supervivencia unos autores son partidarios de tratar únicamente con intención paliativa, mientras que otros preconizan un tratamiento de rescate donde integran la quimioterapia, la cirugía de rescate y la radioterapia $^{9,10}$. Igualmente en nuestro caso fracasaron todas las opciones de tratamiento.

En cuanto a la diseminación a distancia se considera que los sitios más frecuentes de metás- tasis del cáncer vesical son por este orden los ganglios linfáticos, hígado, pulmón y hueso. Sengelov et al. ${ }^{11}$ en un estudio retrospectivo sobre 240 pacientes con cáncer transicional encuentran que en la mayoría de los pacientes se diagnostican las metástasis en los dos primeros años después del diagnóstico. Los sitios más frecuentes de diseminación fueron hueso, ganglios linfáticos, pulmón e hígado; más de la mitad de los pacientes presentaron metástasis en varios órganos simultáneamente. La supervivencia a los 5 años de los pacientes con cáncer vesical infiltrante tratado mediante cistectomía es de aproximadamente el 50\%, debido generalmente a diseminación metastásica microscópica previa a la cirugía. Lógicamente el diagnóstico de la mayoría de las metástasis se realizará en los 2-3 años siguientes al tratamiento. Aunque la cistectomía no debería alterar teóricamente el patrón de diseminación metastásica, existe poca información en la literatura sobre este tema. Sengelov et al. ${ }^{10}$ en 32 pacientes tratados mediante cistectomía con o sin radioterapia, que presentaron metástasis en el seguimiento, encontraron una mayor frecuencia de metástasis en hueso, hígado, pulmón y ganglios linfáticos. Estos pacientes desarrollaron más metástasis hepáticas de forma significativa que los tratados mediante otras técnicas (resección transuretral o radioterapia). Escudero et al. ${ }^{12}$ en 64 pacientes muertos por metástasis encontraron la misma distribución de las metástasis, presentando metástasis óseas el $58 \%$ de los pacientes.

La quimioterapia adyuvante sistémica con esquemas tipo M-VAC, CMV, etc. que conllevan una no despreciable toxicidad, es el único tratamiento que se puede ofrecer a estos pacientes. La respuesta a la quimioterapia dependerá de los factores pronósticos (edad, índice de Karnofsky, lugar de las metástasis, etc.) y de la tolerancia individual de cada paciente. De forma global podemos decir que hasta una tercera parte de los pacientes tratados presentan una respuesta completa aunque dos terceras partes de ellos recidivan. La mediana de supervivencia de los pacientes con respuesta completa llega a 38 meses frente a los 11 meses de los pacientes con respuesta parcial ${ }^{13}$. Hay autores que defienden el tratamiento neoadyuante, en aquellos casos en 
los que se encuentran ganglios en el estudio de diseminación (TAC), como es el caso de la gran serie de 900 cistectomías que presenta Volkmer ${ }^{14}$, su estudio se basa en valorar la supervivencia de estos pacientes sometidos a cistectomía y neoadyuvancia. De estos 900 pacientes, 181 resultaron T0, en un 6,6\% encontraron afectación ganglionar. No encontraron diferencias estadísticamente significativas en la supervivencia en beneficio a los ToNO respecto a los $\mathrm{T}+\mathrm{NO}$, concluyendo que los TO mantienen un riesgo a tener en cuenta de recurrencia tumoral.

Hemos expuesto la hemorragia intraoperatoria como la complicación perioperatoria más frecuente, coincidiendo con la literatura, que añaden a la lista de complicaciones perioperatorias más comunes las cardiovasculares, las infecciosas y el íleo paralítico ${ }^{15}$.

En lo que respecta a las complicaciones originadas por la derivación urinaria cabe destacar: la litiasis, las estenosis ureterointestinales, la retención o incontinencia de orina, la estenosis uretro-neovejiga y las alteraciones metabólicas y nutricionales.

Los pacientes portadores de una derivación urinaria presentan un riesgo aumentado de formación de cálculos urinarios. Se estima una incidencia del 3-4\% en los conductos colónicos, 10$12 \%$ en los conductos ileales, hasta un $20 \%$ en los reservorios cecales continentes y del $2,2 \%$ en las sustituciones vesicales ortotópicas con intestino delgado. Generalmente estas litiasis están formadas por estruvita y fosfato de calcio y su etiología es multifactorial: infección urinaria, alteraciones metabólicas (acidosis metabólica, hipercalciuria, hipocitraturia e hiperoxaluria), estanqueidad de la orina, producción de moco en el segmento intestinal y presencia de cuerpos extraños (suturas metálicas) ${ }^{16}$.

Entre las complicaciones de la anastomosis uretero-intestinal destacan la aparición de fistula urinaria y la estenosis de la unión. La incidencia de estenosis uretero-intestinales admitida de forma clásica en la literatura oscila entre el $4 \%$ y el $8 \%$. Los factores etiológicos implicados en el desarrollo de una estenosis uretero-intestinal son múltiples, los más importantes son: falta de aposición mucosa-mucosa, tensión en la anastomosis e isquemia ureteral o intestinal ${ }^{17,18}$.
Debido a que la estenosis uretero-intestinal suele ser secundaria a defectos en la técnica quirúrgica de disección (ureteral o intestinal) o anastomosis, cabría esperar que se desarrollara con cierta rapidez. Se ha considerado clásicamente que las estenosis uretero-intestinales se diagnostican en los dos primeros años después de la cirugia $^{15}$. En la serie de Meretyk et al. ${ }^{17}$, de pacientes con estenosis uretero-intestinal tratadas mediante procedimientos endourológicos, el tiempo medio de aparición de la estenosis fue de 32,7 (1-72) meses, aunque sólo la mitad de los pacientes se diagnosticaron en los dos años siguientes a la cirugía y el $40 \%$ pasados cuatro años de la intervención quirúrgica. En la serie de Jahnson y Pedersen ${ }^{19}$ el tiempo medio desde la cirugía hasta el diagnóstico de la estenosis fue de 29 (2-108) meses, diagnosticándose el 79\% de las mismas en los primeros 5 años. En nuestra experiencia, las técnicas anti-reflujo presentan una incidencia algo mayor de estenosis, por lo que es necesario un control de los pacientes a largo plazo. En la serie publicada por P. Valdivia et al. ${ }^{20}$ sobre 80 sustituciones vesicales tipo Hautmann realizadas en nuestro Servicio entre 1990 y 1998, se analizan las estenosis uretero-intestinales encontrando un 5,6\% en los casos en los que se utilizó la técnica del surco mucoso de Le Duc, y un $18,5 \%$ cuando se empleó la técnica del túnel seroso extramural de Abol-Enein.

La aparición de alteraciones metabólicas y nutricionales después de una derivación urinaria dependerá de la función renal, del segmento intestinal utilizado y del tiempo de contacto entre la orina y el intestino. En los pacientes en los que se utilizó el íleon o el colon presentan una acidosis metabólica hiperclorémica, más frecuente en los pacientes con sustitución vesical ortotópica o bolsas continentes que en los pacientes con conducto ileal o colónico. Aunque casi todos presentaran datos analíticos de acidosis muy pocos presentarán síntomas clínicos. La fistula urinaria suele aparecer dentro de los primeros 7-10 días tras la cirugía, siendo su incidencia entre un 3\% y un 9\%, reduciéndose esta con la utilización de catéteres ureterales durante el postoperatorio inmediato. Una fuga de orina provoca una reacción inflamatoria perianastomótica con fibrosis posterior, lo que puede causar obstrucción y estenosis posterior ${ }^{8}$. 
Después de analizar de una manera más global las indicaciones y complicaciones de las cistectomías, pasemos a comparar nuestros resultados con las escasa series existentes en la literatura. Thrasher ${ }^{21}$, del grupo de Paulson, presentaron su serie de 66 casos de pTO en 433 cistectomías (15,2\%) entre 1969 y 1990. Analizando sus datos epidemiológicos, son similares a los de nuestra serie, como edad media de 64 años, porcentaje mucho más elevado de varones, etc... asî como los aspectos evolutivos, ya que presentan una afectación ganglionar del 9\%. Sin embargo, a la hora de los resultados concluye que no encuentran mejor supervivencia en los pTO y la supervivencia de estos viene determinada por el estadio clínico inicial. Del mismo series largas de cistectomía, como la de $\operatorname{Stein}^{22,23}$ que concluyen que la afectación ganglionar y la recurrencia viene determinada por el estadio previo.

Por otra parte Ansong ${ }^{24}$ refuta el axioma de Thrasher destacando la mayor supervivencia de éstos.

En la literatura son pocas las series de pTO, normalmente se presentan análisis de series de carcinoma vesical infiltrante como las presentadas por Escudero ${ }^{12,25}$ o Lee $^{26}$ donde se centran en el beneficio de la cistectomía parcial y sobre todo en estos casos donde no hayan tumor en la pieza. $\mathrm{El}$ tratamiento del tumor vesical infiltrante suscita en muchas ocasiones una gran polémica y más en estos pacientes pTO, donde toman un mayor papel los defensores de la conservación vesical, como la serie de 217 casos publicada por $\mathrm{Herr}^{27}$ o la propia de Solsona ${ }^{5}$, apoyados en la morbimortalidad de la cistectomía.

En nuestro estudio no hemos comparado la supervivencia de los pTO con otros estadios, es meramente un estudio descriptivo de los pTO. Tampoco entramos en la discusión del tratamiento más adecuado del carcinoma vesical infiltrante, pero si mencionar que la cistoprostatectomía radical contribuiría al control local de la enfermedad, a la vez que permitiría una correcta estadificación patológica en los pTO posibilitando la aplicación de tratamiento sistémico asociado ante $\mathrm{pN} 1-2$.

$\mathrm{Al}$ estudiar las variables predictivas de supervivencia en el cáncer vesical se observa la falta de grandes progresos. En el análisis multivariante, el estadio tumoral o el estado ganglionar se presentan como los datos de mayor importancia pronóstica. Solsona ${ }^{28,29}$ en su serie de 298 pacientes tratados sólo con cistectomía distingue grupos de riesgo pronóstico también según la afectación ganglionar, el estadio y la infiltración prostática. Mientras otros estudios contemplan que la edad, el sexo o la raza no son factores consistentes en la predicción de la supervivencia ${ }^{1}$.

\section{CONCLUSIONES}

En nuestra experiencia los tumores uroteliales pTO presentan un periodo libre de enfermedad prolongada (mediana de 180 meses).

Los factores de riesgo asociados a un menor PLE son alto grado de diferenciación (G3, 116 meses), la infiltración de capas profundas en la RTU (T3, 32 meses) y la afectación ganglionar (pN+ 45 meses).

\section{REFERENCIAS}

1. Blanco FJ, Herr HW. Cáncer vesical infiltrante: manejo basado en evidencia clínica. Angulo J Berenguer A, editors.Cáncer de vejiga. Historia natural. Biología. Terapéutica. $1^{a}$ edición. Madrid: Luzán 5. 2004;345-353.

2. Palapattu GS, Shariat SF, Karakiewick PI, Bastian PJ, Rogers CG, Amiel G, et al. Cancer Specific Outcomes in Patients With PTO Disease Following Radical Cystectomy. J Urol. 2006(5);175:1645-1649.

3. Oosterlink W, Lobel B, Jakse G, Malmström PU, Stöckle M, Sternberg C. "Guidelines on BLADDER cancer". in: EAU's Guidelines for Urology. Edited for the E.A.U. Healthcare Office. European Association of Urology. Drukkerij Gelderland bv, Arnhem, The Netherlands. 2001.

4. Angulo JC, López JI. Definición y manejo del cáncer vesical superficial de alto riesgo. Revisiones en Urología 2002; Vol III (1):12-19.

5. Solsona E, Iborra I, Rubio J, Casanova J, Dumont R, Monros JL. Late oncological ocurrences following radical cystectomy in patients with bladder cancer. Eur Urol. 2003;43(5):489-494.

6. Solsona E, Iborra I, Ricos JV, Monros JL, Casanova J, Calabuig C. Feasibility of transurethral resection for muscle infiltrating carcinoma of the bladder: long-term followup of a prospective study. J Urol. 1998;159(1):95-98.

7. Montie JE: Follow-Up After Cystectomy For Carcinoma Of The Bladder. Urol Clin North Am 1994;21(4):639-643.

8. Herranz Amo F. Seguimiento de los pacientes con cáncer vesical tratados mediante cistectomía radical. Actas Urol Esp. 2000;24(2): 144-154.

9. Pisters LL, Westney L. The Management of Locally Recurrent Invasive Bladder Cancer Following Radical Cystectomy. Semin Urol Oncol. 1996;14(2):112-119.

10. Jiménez Cruz JF, Vera Donoso CD, Sanz Chiniesta S. La Recidiva Pélvica Post-Cistectomía. Actas Urol Esp. 1996; 20(6):593-596. 
11. Sengelov L, Kamby C, Maase HVD. Pattern of metastases in relation to characteristics of primary tumor and treatment in patients with disseminated urothelial carcinoma. J Urol. 1996;155(1):111-114.

12. Escudero A, Fernández E, Jiménez M, Maganto E. Cistectomía parcial como tratamiento del carcinoma transicional infiltrante de vejiga. Act Urol Esp. 1997;21(6):572589 .

13. Sternberg CN, Arena MG, Calabresi F, De Carli P, Platania A, Zeulim M, et al. Metotrexato, vinblastina, doxorrubicina y cisplatino para el carcinoma urotelial infiltrante: eficacia y patrones de respuesta. Practical cases in Urology. Cancer treatment stage by stage (edición española) 1995;3: 115-133.

14. Volkmer BG, Kuefer R, Bartsch G Jr, Straub M, De Petriconi R, Gschwend JE, et al . Effect of a pTO cystectomy specimen without neoadjuvant therapy on survival. Cancer. 2005;104(11):2384-2391.

15. Quek ML, Stein JP, Daneshmand S, Miranda G, Thangathurial D, Roffey P, et al. A critical analysis of perioperative mortality from radical cystectomy. J Urol. 2006; 175(3pt1):886-889.

16. Regalado R, Huguet J, Errando C, Lima BX, Chechile G, Villavicencio $\mathrm{H}$ : Sustitución vesical ortotópica: III. Resultados funcionales y complicaciones en los pacientes con neovejiga ileal tipo Studer. Arch Esp Urol. 1997;50 (3):234-241.

17. Meretyk S, Clayman Rv, Kavoussi Lr, Kramolowsky Ev, Picus Dd. Endourological treatment of ureteroenteric anastomotic strictures: longterm followup. J Urol. 1991; 145(4):723-727.

18. Tolhurst SR, Rapp DE, O`Connor RC, Lyon MB, Orvieto MA, Steinberg GD. Complications after cystectomy and urinary diversion in patients previously treated for localized prostate cancer. Urology. 2005;66(4):824-829.

19. Jahnson S, Pedersen J. Cistectomía y derivación urinaria durante veinte años. Complicaciones e implicaciones metabólicas. Eur Urol (edición española) 1994;8:607-613.

20. Valdivia P, Gil MJ, Gil P, Liédana JM, Roncalés A, Rioja LA, Hassan Abol-Enein. Reimplantation. Analysis Of Our Experience. Póster XVI Congreso Europeo de Urología, Ginebra, 2000.
21. Thrasher JB, Frazir HA, Robertson JE, Paulson DF. Does a stage pTO cystectomy specimen confer a survival advantage in patients with minimally invasive bladder cancer?. J Urol. 1994;152(2pt1):393-396.

22. Stein JP, Lieskovsky G, Cote R, Groshen S, Feng AC, Boyd $\mathrm{S}$, et al. Radical cystectomy in the treatment of invasive bladder cancer: long-term results in 1.054 patients. J Clin Onc. $2001 ; 19(3): 666-675$.

23. Stein JP, Skinner DG. Results with radical cystectomy for treating bladder cancer: a "reference standard" for highgrade, invasive bladder cancer.BJU Int. 2003;92(1):12-17.

24. Amsong K. Re. Does a stage pTO cystectomy specimen confer a survival advantage in patients with minimally invasive bladder cancer?. J Urol. 1995;153(3 pt1):751.

25. Escudero A, Fernández E, Jiménez M, Maganto EM. Análisis de 34 casos de carcinoma infiltrate de vejiga tratados con cistectomía parcial exclusivamente. Arch Esp Urol. 1996;49(4):349-364.

26. Lee SE, Jeong IG, Ku JH, Kwak C, Lee E, Jeons JS. Inpad of transurethral resection of bladder tumor: Analysis of cystectomy specimens to evaluate for residual tumor. Urol. 2004;63(5):873-877.

27. Herr HW. Conservative managemrnt of muscle-infiltration bladder cancer: prospective experience. J Urol.1987;138(5): 1162-1165.

28. Solsona E, Iborra I, Dumont R, Rubio J, Casanova J, Almenar S. Risk groups in patients with bladder cancer treated with radical cystectomy: stadistical and clinical model improving homogeneity. J Urol. 2005;174(4 pt1): 1226-1230.

29. Solsona E, Iborra I, Rubio J, Casanova J, Almenar S. R. The optium timing of radical cystectomy for patients with recurrent high-risk superficial bladder tumour. BJU Int. 2004;94(9): 1258-1262.

Dra. E. Mallén Mateo

E-mail: evamallen@mixmail.com

(Trabajo recibido el 18 de abril de 2006) 